INPLASY

PROTOCOL

To cite: Wang et al. Various doses of xuebijing injections for sepsis treatment: $A$ protocol for systematic review and meta-analysis. Inplasy protocol 202210082. doi: 10.37766/inplasy2022.1.0082

Received: 16 January 2022

Published: 16 January 2022

Corresponding author: Qianfei Wang

1025903923@qq.com

Author Affiliation:

Graduate School of Hebei

University of Chinese

Medicine.

Support: Hebei Provincial

Government Fu.

Review Stage at time of this submission: The review has not yet started.

Conflicts of interest:

None declared.

\section{Various doses of xuebijing injections for sepsis treatment: A protocol for systematic review and meta-analysis}

Wang, QF1; Chen, FQ2; Liu, D3; Liu, Y4; Tao, YL5; Mei, JQ6.

Review question / Objective: This study is intended to evaluate the efficacy of various doses of xuebijing injections for sepsis treatment.

Condition being studied: Sepsis is characterized by high incidence rate, high mortality, and difficulty in curing. About 14000 people worldwide die of sepsis and its complications every day, which seriously affects the quality of human life and poses a great threat to human health. Xuebijing has obvious anti-inflammatory and promoting blood circulation and removing blood stasis effects. At present, it has been widely used in the treatment of critical diseases and sepsis in China.This study aimed to systematically review the effectiveness of xuebijing in the treatment of sepsis.

INPLASY registration number: This protocol was registered with the International Platform of Registered Systematic Review and Meta-Analysis Protocols (INPLASY) on 16 January 2022 and was last updated on 16 January 2022 (registration number INPLASY202210082).

\section{INTRODUCTION}

Review question / Objective: This study is intended to evaluate the efficacy of various doses of xuebijing injections for sepsis treatment.

Condition being studied: Sepsis is characterized by high incidence rate, high mortality, and difficulty in curing. About 14000 people worldwide die of sepsis and its complications every day, which seriously affects the quality of human life and poses a great threat to human health. Xuebijing has obvious anti-inflammatory and promoting blood circulation and removing blood stasis effects. At present, it has been widely used in the treatment of 
critical diseases and sepsis in China.This study aimed to systematically review the effectiveness of xuebijing in the treatment of sepsis.

\section{METHODS}

Search strategy: The search terms include: xuebijing、Various doses xuebijing, Low dose xuebijing, medium dose xuebijing, high doses xuebijing, sepsis. The combined method of MeSH term and free words was used for search. The complete PubMed search strategy is summarized in Table 1. The search strategies of the Chinese database and other databases were similar.

Participant or population: Inclusion: (1)Adults with sepsis (as diagnosed using any recognised diagnostic criteria). (2) aged 18-80 years. (3) signing informed consent.Exclusion: (1) not meeting the diagnostic criteria.(2)Adolescents (under 18 years of age) and elderly people (over 80 ).

Intervention: 1. conventional treatment and $50 \mathrm{ml} /$ time, twice / day xuebijing2. conventional treatment and $100 \mathrm{ml} /$ time, twice / day xuebijing3. conventional treatment and $100 \mathrm{ml} /$ time, 4 conventional treatment and $100 \mathrm{ml} /$ time, twice / day xuebijing5. conventional treatment and $100 \mathrm{ml} /$ time, 4 times/ day xuebijing.

Comparator: 1.conventional treatment2. conventional treatment 3 . conventional treatment 4. conventional treatment and $50 \mathrm{ml} /$ time, twice / day xuebijing 5 . conventional treatment and $100 \mathrm{ml} /$ time, twice / day xuebijing.

Study designs to be included: Randomized clinical trials.

Eligibility criteria: Diagnosis criteria include 《Consensus of Chinese experts on early prevention and blocking of sepsis》 and 《Surviving Sepsis Campaign: international guidelines for management of sepsis and septic shock 2021, interpretation and expectation》. Language is in Both Chinese and English and it is RCTs. The observation group was treated with xuebijing. nonRCTs, dissertations, reports, conferences, case reports, and incomplete, duplicate literature.will beexcluded.

Information sources: CNKI, Wan Fang, VIP, PubMed, Cochrane Library, WOS,Embase.

Main outcome(s): WBC, CRP, PCT and coagulation (APTT, Pt, TT and FIB).

Additional outcome(s): IL-6, IL-18 and TNFa, D-dimer.

Quality assessment / Risk of bias analysis: Document quality evaluation: Cochrane system evaluation manual (version: 5.0.2) is used to evaluate the quality of the included documents. Each item is evaluated in three categories: "high bias risk", "unclear" and "low bias risk".

Strategy of data synthesis: The data collection andanalysis was conducted as follows:Applying the EndnoteX7 software to manage the included references. Data collection: The outline of literature collection was formulated, including author, year, sample size of two groups, treatment method, treatment time, blind method, outcome index, etc. Two authors searched the literature, collected relevant data, and checked all the data. If there is any inconsistency between the included data and the project opinions, a third person shall be involved to solve it.

Subgroup analysis: Subgroup analysis can be carried out according to the patient's age and dose.

Sensitivity analysis: When more than 10 articles were included, a funnel chart should be drawn to judge the publication bias.

Country(ies) involved: China.

Keywords: xuebijing, sepsis, systematic review.

Contributions of each author:

Author 1 - qianfei wang.

Author 2 - fenqiao chen. 
Author 3 - dong liu.

Author 4 - yuan liu.

Author 5 - yulei tao.

Author 6 - jianqiang mei. 